\title{
The New Digital Divide For Digital Biomarkers
}

\author{
John Torous ${ }^{\mathrm{a}, \mathrm{b}} \quad$ Jorge Rodriguez ${ }^{\mathrm{b}}$ Adam Powell $^{\mathrm{c}}$ \\ a Department of Psychiatry, Beth Israel Deaconess Medical Center, Harvard Medical School, \\ Boston, MA, USA; b Division of Clinical Informatics, Beth Israel Deaconess Medical Center, \\ Harvard Medical School, Boston, MA, USA; ${ }^{C}$ Payer+Provider Syndicate, Boston, MA, USA
}

\author{
Keywords \\ Digital biomarkers $\cdot$ Smartphones $\cdot$ Methods $\cdot$ Technology
}

\section{Abstract}

As smartphone and sensors continue to become more ubiquitous across the world, digital biomarkers have emerged as a scalable and practical tool to explore disease states and advance health. However, as the digital divide of access and ownership begins to fade, a new digital divide is emerging. Who are the types of people who own smartphones or smart watches, who are the types of people who download health apps or partake in digital biomarker studies, and who are the types of people who are actually active with digital biomarker apps and sensors - the people providing the high-quality and longitudinal data that this field is being founded upon? Understanding the people behind digital biomarkers, the very people this emerging field aims to help, may actually be the real challenge as well as opportunity for digital biomarkers.

Unaddressed mental health issues are now a leading global cause of disability and suffering. While mortality from cardiovascular diseases and cancer is beginning to decline, suicide rates are increasing [1]. Limited global availability of effective mental health treatments, a lack of objective measures of response to treatment, and a lack of specificity in mental health diagnoses remain barriers in advancing patient outcomes. Thus, there has been increasing interest in new solutions and tools to guide both research and treatment. Digital biomarkers, obtained directly through the use of patients' personal smartphones and wearable sensors, have generated significant attention as a novel means for capturing real-time, in vivo, longitudinal behaviors and self-assessments related to mental health [2]. While no 
Torous et al.: Digital Divide for Digital Biomarkers

Fig. 1. A conceptual schematic of digital biomarkers. The blue diamonds represent individual digital biomarkers and sample raw data used to generate them. The red box represents composite digital biomarkers and the yellow diamonds traditional basic science biomarkers.

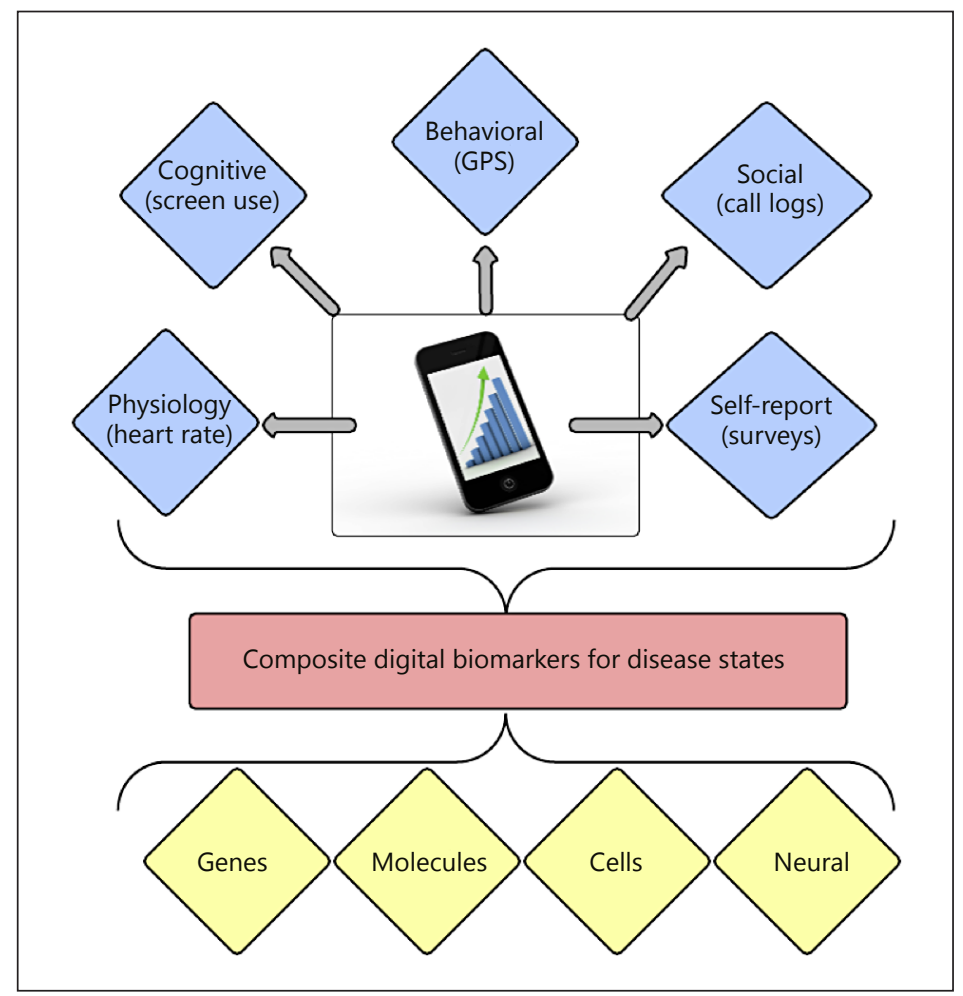

major organization has precisely defined mental health digital biomarkers, in part because of the nascence and rapid expansion of the topic, Figure 1 provides an overview of the types of data which could be included in a definition. Each blue diamond in Figure 1 represents a raw digital biomarker. It is possible to combine several raw biomarkers into a composite biomarker for a disease state, as represented by the red box. These composite digital biomarkers offer immediate clinical applications and may also serve as a means to better interpret the findings of basic science research, as indicated by the yellow diamonds. For example, researchers are actively investigating whether smartphone-based GPS data may serve as a digital biomarker for depression [3], as those suffering from the illness may exhibit reduced and altered daily movements. Others have suggested that digital biomarkers from call and text message logs may be useful in identifying transitions to mania in bipolar disorder [4]. The low cost and scalability of smartphone- and app-based digital biomarkers facilitates a potential wealth of behavioral, social, environmental, and even physiological data that was previously unobtainable [5-7].

The surge in interest of digital biomarkers for mental health is related to several converging factors. Increasing ownership of mobile devices has largely closed the digital divide of access to connected technologies. Currently, $77 \%$ of US adults own a smartphone, double the 35\% ownership rate in 2011 [8]. While it was claimed in the past that those with mental illnesses may not own or be interested in using digital devices as part of their mental healthcare, recent evidence has shown that, like the rest of the global population, those with mental illness own smartphones and are interested in health apps [9]. While ownership rates do vary by race, education, and income, smartphones and health apps are of interest across the socioeconomic boundaries [10].

This interest is currently met by thousands of apps and tools for mental health released in the Apple and Android stores [11]. New digital therapy companies are receiving multi- 
Torous et al.: Digital Divide for Digital Biomarkers

million dollar investments to create digital biomarkers through apps and wearable sensors. Apple's HealthKit and Android's Research Stack framework have further reduced the barriers to developing intuitive, patient-centered apps that may serve as digital biomarkers. Thus, patients now have access to both the devices and apps necessary to transform their phone into a source of digital biomarkers and unlock previously inaccessible information about mental health.

Despite this potential, to date research on digital biomarkers for mental health has produced mixed results [12] with no definitively reproducible findings. We propose that while the first digital divide of access to mobile technology has closed for mental health patients, in its place, there is now a new digital divide, in which usability rather than access hampers the potential of digital biomarkers.

To put it in simpler terms: who is actually using these mental health apps to produce digital biomarker data? Despite the often-posited potential, there is little discussion or data on user demographics. Without an understanding of which patients are using apps, both the personal- and population-level benefits of mobile and connected mental health technology may be lost. The first step in the user-centered design process is understanding the context of use. This step is challenging to accomplish, as app vendors are not publishing data on their users. In some cases, privacy laws may make it illegal for hospitals, software companies, or app developers to share certain types of data about their users [13]. It is also likely that the demographics of healthcare app users are evolving over time, and are not consistent across use cases; for instance, it is doubtful that men frequently use ovulation tracking apps. Although such data are not available, it is possible to glean some information from reviewing smartphone ownership data and prior clinical studies. Understanding the current use of digital technology for mental health is fundamental to realizing the potential of this technology, as even the best biomarker is useless if unused.

\section{Health App Use in the Literature}

Although few studies have examined health app user demographics, several have provided some insights. One study conducted via an online survey of 1,604 US adults reported that $58 \%$ of participants had downloaded a health app. The users in this study tended to be younger, be Latino, have higher income, have greater than high school education, and have a higher body mass index than the national average. There was no association between downloading a health app and gender or having a chronic disease [14]. Considering who partakes in app clinical studies, there is no clear consensus on which demographics may be over or underrepresented, although there is some evidence that those aged $<30$ years are often a large competent of the sample in app studies $[15,16]$. At this point, it remains unclear why certain people choose to partake in app research or share their app data. Outside of studies, interest in an app or even downloading a health app does not guarantee use, as seen in one survey study, where $85 \%$ of subjects expressed interest in using health apps, although only $17 \%$ had actually ever used one [10]. When actual users are studied, a common limitation of app-focused studies is the use of crowdsourcing to recruit individuals from the Internet. When crowdsourcing is used, the study population consists of people without verified diagnoses. While such recruitment methods facilitate a large sample size, they have an inherent selection bias as they include participants who are both online and interested in taking surveys. Thus, crowdsourced studies may not produce findings which are representative of the general population. To date, there has not been a systematic review of the demographics of people included in app-related research. Ensuring that the voices of all patients, even those not digitally connected, are represented will help us get a clearer understanding of true patient needs. 


\section{App Adherence}

Data on app downloaders is too insufficient to understand the demographics of app users, as people frequently disengage from apps after downloading them. A study of users of the PTSD Coach app revealed that while the app has been downloaded 166,861 times the last three years, only $14 \%$ of users opened the app 1 day after downloading it, and only just over $4 \%$ were using the app 1 year later [17]. While this study did not collect data on whether users had PTSD or not, another study suggested that those with chronic illness may be less adherent to apps compared to a healthy control population. The study found that controls were $76 \%$ adherent, while those with chronic illness were only $16 \%$ adherent when asked to use a diet app and 4 health-tracking devices during a 4-week period [18]. Understanding the degree to which patients will maintain adherence to apps as they become more ill is another topic that is understudied. Similar to medication adherence, app adherence will benefit from gaining an understanding of barriers to use, thus driving the design of optimal digital tools to provide care. While studies on why patients engage with apps may not offer immediate new diagnostic data or treatment tools that can serve as digital biomarkers, understanding engagement is the foundation for the success of all health apps.

\section{App Barriers}

Among those with mental illness, use data have been rarely reported or studied, although qualitative studies are beginning to answer why app use may be low. In the adeptly named paper, "It Feels Different from Real Life," the authors conducted a thematic analysis of user reviews of mental health apps on various online forums and app stores. They report that current limitations of mental health apps include: (1) not providing enough emotional support, (2) distracting users from real-life challenges, (3) causing users to not seek in-person care or peer support, and (4) receiving self-reported symptoms from users which differ from what they would report in person [19]. One phone survey of Australian youth assessed willingness to adopt app-based versus traditional mental health services and concluded that the desire for app-based services is low and that respondents questioned the efficacy of such services [20]. End user interest and concerns should be studied during the health app design process to both inspire features and reduce barriers to access. As referenced above, while some mental health apps undergo research evaluation [21,22], the vast majority does not and little is known about their safety or efficacy.

Finally, any lack of patient or clinician trust in health apps, be it trust that the apps are effective or trust that the apps protect privacy, is a barrier that cannot be ignored [23]. Instead of focusing only on the success of apps, learning from their failures will help us create new digital tools that can overcome barriers and increase use.

\section{Conclusion}

While the potential of digital biomarkers for mental healthcare has become increasingly clear, there is still a lack of clarity surrounding who is using them and how they are being used. Although the digital divide in access to information technology has largely dissipated, a new divide has emerged between the creators and users of digital services. The first step in developing digital biomarkers for advancing mental health research and treatment is to understand the needs of patients. Potential next steps include developing more ecological and anthropological research as well as addressing the lack of usability, trust, and evidence which 
Torous et al.: Digital Divide for Digital Biomarkers

plagues many apps. Bridging the new digital divide is feasible if we focus less on the technology creating digital biomarkers and more on the people actually using technology to quantify and share their experiences with illness.

\section{Conflict of Interest Statement}

The authors declare no conflicts of interest. Dr. Torous is supported by the Myrtlewood Foundation and a T15 Training Grant (4T15LM007092-25) from the National Library of Medicine.

\section{References}

1 Centers for Disease Control and Prevention. Increase in Suicide in the United States, 1999-2014. http://www. cdc.gov/nchs/products/databriefs/db241.htm\#suicide_rates (accessed January 10, 2017).

$\checkmark 2$ Torous J, Onnela JP, Keshavan M: New dimensions and new tools to realize the potential of RDoC: digital phenotyping via smartphones and connected devices. Transl Psychiatry 2017;7:e1053.

-3 Saeb S, Zhang M, Karr CJ, Schueller SM, Corden ME, Kording KP, Mohr DC: Mobile phone sensor correlates of depressive symptom severity in daily-life behavior: an exploratory study. J Med Internet Res 2015;17:e175.

-4 Faurholt-Jepsen M, Vinberg M, Frost M, Christensen EM, Bardram JE, Kessing LV: Smartphone data as an electronic biomarker of illness activity in bipolar disorder. Bipolar Disord 2015;17:715-728.

5 Kenny R, Dooley B, Fitzgerald A: Ecological momentary assessment of adolescent problems, coping efficacy, and mood states using a mobile phone app: an exploratory study. JMIR Ment Health 2016;3:e51.

6 Beiwinkel T, Kindermann S, Maier A, Kerl C, Moock J, Barbian G, Rössler W: Using smartphones to monitor bipolar disorder symptoms: a pilot study. JMIR Ment Health 2016;3:e2.

-7 Ben-Zeev D, Scherer EA, Wang R, Xie H, Campbell AT: Next-generation psychiatric assessment: using smartphone sensors to monitor behavior and mental health. Psychiatr Rehabil J 2015;38:218.

8 Smith A: Record shares of Americans now own smartphones, have home broadband. Pew Research Center, 2017.

9 Torous J, Chan SR, Tan SY, Behrens J, Mathew I, Conrad EJ, Hinton L, Yellowlees P, Keshavan M: Patient smartphone ownership and interest in mobile apps to monitor symptoms of mental health conditions: a survey in four geographically distinct psychiatric clinics. JMIR Ment Health 2014;1:e5.

10 Ramirez V, Johnson E, Gonzalez C, Ramirez V, Rubino B, Rossetti G: Assessing the use of mobile health technology by patients: an observational study in primary care clinics. JMIR mHealth uHealth 2016;4:e41.

-11 Powell AC, Torous J, Chan S, Raynor GS, Shwarts E, Shanahan M, Landman AB: Interrater reliability of mHealth app rating measures: analysis of top depression and smoking cessation apps. JMIR mHealth uHealth 2016;4:e15.

$\checkmark 12$ Torous J, Kiang MV, Lorme J, Onnela JP: New tools for new research in psychiatry: a scalable and customizable platform to empower data driven smartphone research. JMIR Ment Health 2015;3:e16.

13 Donker T, Petrie K, Proudfoot J, Clarke J, Birch MR, Christensen H: Smartphones for smarter delivery of mental health programs: a systematic review. J Med Internet Res 2013;15:e247.

14 Bakker D, Kazantzis N, Rickwood D, Rickard N: Mental health smartphone apps: review and evidence-based recommendations for future developments. JMIR Ment Health 2016;3:e7.

15 Examining Oversight of the Privacy and Security of Health Data Collected by Entities Not Regulated by HIPAA. https://www.healthit.gov/buzz-blog/privacy-and-security-of-ehrs/examining-oversight-privacy-securityhealth-data-collected-entities-not-regulated-hipaa/ (accessed January 10, 2017).

16 Krebs P, Duncan DT: Health app use among US mobile phone owners: a national survey. JMIR mHealth uHealth 2015;3:e101.

17 Owen JE, Jaworski BK, Kuhn E, Makin-Byrd KN, Ramsey KM, Hoffman JE: mHealth in the wild: using novel data to examine the reach, use, and impact of PTSD Coach. JMIR Ment Health 2015;2:e7.

18 Shaw RJ, Steinberg DM, Bonnet J, et al: Mobile health devices: will patients actually use them? J Am Med Inform Assoc 2016;23:462-466.

19 de Alva F, Wadley G, Lederman R: It feels different from real life: users' opinions of mobile applications for mental health 2015;598.

20 Schuster L, Drennan J, Lings I: Understanding consumers' decisions to adopt technology-enabled transformative services. Service Ind J 2015;35:846-864.

-21 Anguera JA, Jordan JT, Castaneda D, Gazzaley A, Areán PA: Conducting a fully mobile and randomised clinical trial for depression: access, engagement and expense. BMJ Innov 2016;2:14-21.

-22 Mohr DC, Tomasino KN, Lattie EG, Palac HL, Kwasny MJ, Weingardt K, Karr CJ, Kaiser SM, Rossom RC, Bardsley LR, Caccamo L: IntelliCare: an eclectic, skills-based app suite for the treatment of depression and anxiety. J Med Internet Res 2017;19:e10.

23 Torous J, Roberts LW: Needed innovation in digital health and smartphone applications for mental health: transparency and trust. JAMA Psychiatry 2017;74:437-438. 